\title{
Growth and Developmental Responses of Seeded and Seedless Grape Berries to Shoot Girdling
}

\author{
Xiuren Zhang, ${ }^{1}$ Guoguang Luo, ${ }^{2}$ Ronghui Wang, ${ }^{3}$ and Jing Wang ${ }^{1}$ \\ Department of Horticulture, China Agricultural University, Beijing 100094 \\ David G. Himelrick ${ }^{4}$ \\ Department of Horticulture, Louisiana State University, Baton Rouge, LA 70803-2120
}

\begin{abstract}
ADDITIONAL INDEX wORDs. growth dynamics, veraison, soluble solids, titratable acidity, pectic substance, plant hormones, Vitis vinifera

ABstract. The relationship of assimilate supply to grape (Vitis vinifera L.) berry growth and development was studied with a seeded ('Kyoho') and a seedless ('Seedless Wuhehong') cultivar. A single shoot girdling between the second and third nodes below the basal cluster at the end of Stage I of berry growth shortened Stage II (the lag phase) of 'Kyoho' grape berries by 10 days, and eliminated Stage II in 'Seedless Wuhehong' grape berries. Double shoot girdling between the second and third nodes below the basal cluster and above the upper cluster, respectively, at the same time at the end of Stage I, advanced Stage II by 3 days in both cultivars. Normal accumulation of dry weight in the 'Kyoho' grape berry is in a double sigmoidal pattern, but it became a single sigmoidal pattern in response to a single basal girdling. The highest percent moisture in berries was at 20 days after full bloom. Rapid changes in berry pectin substances lagged behind those of soluble solids and titratable acidity, and behind the onset of berry softening at veraison in 'Kyoho', but not in 'Seedless Wuhehong', for which the three processes were concurrent. It is suggested that the slow growth of the berries during Stage II is a result of a decrease in the rate of water accumulation on a whole berry basis and a decrease in accumulation of dry matter in the skin and flesh (pericarp) due to assimilate competition within grapevines and within berries. The relationships between levels of endogenous hormones (IAA, $\mathrm{GA}_{3}$, zeatin, zeatin riboside, and $\mathrm{ABA}$ ) and berry growth were also studied with 'Kyoho' grapes. The results showed that the slow growth of grape berries during Stage II was associated with assimilate competition between the skin-flesh (pericarp) and seeds, and with peak shifts of concentrations of IAA, $\mathrm{GA}_{3}$, zeatin and zeatin riboside. Changes in $\mathrm{ABA}$ levels were closely associated with ripening and senescence during late Stage III.
\end{abstract}

Grape (Vitis vinifera L.) berries exhibit double sigmoidal growth curves. According to Coombe $(1976,1989,1992)$, Coombe et al. (1987), and many other researchers (Cawthon and Morris, 1982; Geza et al., 1984; Staudt et al., 1986; Xu et al., 1995). Stage I of berry growth is characterized by a rapid increase in berry volume, a high rate of cell division in the pericarp, and completion of nucellus and endosperm growth with seeds almost fully grown. In Stage II, there is a decrease in growth rate, the level of auxin reaches a maximum, and the level of ABA begins to increase. In Stage III, there is a change in color and texture of the berries, a further increase in volume due to accumulation of sugar and water, and further increase in $\mathrm{ABA}$ concentration to a maximum at the beginning of the phase before a decrease. Many attempts have been made to describe and explain the mechanisms that control the changes in fruit growth rate, including hypotheses that developmental changes in the pericarp are linked to changes in seeds or result from competition with vegetative growth (Matsui et al., 1985; Niimi and Torikata, 1979; Novello et al., 1999; Staudt et al., 1986). Several plant hormones may play an important role in the growth and development of grape berries (Brenner et al., 1989; Cawthon and Morris, 1982; Inaba et al., 1976; Niimi et al., 1977; Pandey and Singh, 1989; Tromp, 1989). Much attention

Received for publication 5 Aug. 2002. Accepted for publication 11 Dec. 2002. This research was funded by China's National Scientific Foundation Committee (grant 3880604). This paper is a portion of a thesis submitted by Xiuren Zhang in partial fulfillment of MSc degree requirement. The authors are very much grateful to Prof. Xu, Xuefeng for his assistance with this research.

${ }^{1}$ Current address: Department of Plant Biology and Boyce Thompson Institute for Plant Research, Cornell University, Ithaca, NY 14850.

${ }^{2}$ To whom correspondence should be addressed; email gluo@public.cau.edu.cn. ${ }^{3}$ Department of Soil Science and Plant Nutrition, China Agricultural University, Beijing 100094.

${ }^{4}$ To whom correspondence should be addressed; email dhimelrick@agctr.lsu.edu. has been paid to the ripening process with the suggestion that the auxin-ABA relationship might be involved in the hormonal regulation of veraison and ripening of grape berries.

Although many studies have been done on double sigmoidal growth of grape berries, the mechanisms leading to the double sigmoidal growth pattern remain unclear. For better understanding of these mechanisms, we investigated the growth kinetics and related physiological and biochemical processes occurring during the berry growth, and the relationship among assimilate competition, double-sigmoid growth kinetics and endogenous hormone levels in grape berries. Considering that cane- or trunk-girdling has been reported to affect berry weight and advance fruit maturity by changing the assimilate translocation and distribution in grapevines (Harrel and Williams, 1987; Peruzzo, 1994; Zabadal, 1992), we designed two shoot girdling treatments to change indirectly the assimilate supply to berries of two cultivars, one seeded ('Kyoho') and one seedless ('Seedless Wuhehong'), to help understand the role of assimilate supply in berry development. 'Seedless Wuhehong' is stenospermocarpic, with normal pollination and fertilization followed by embryo abortion. Some traces of aborted seeds can be seen in berries.

\section{Materials and Methods}

Plant material. Grapevines of two cultivars, 'Kyoho' (Vitis labrusca L. x Vitis vinifera L.) and 'Seedless Wuhehong' (Vitis vinifera $\mathrm{L}$.), were used in this experiment. They were grown in the vineyards of Baijiangtong County, Haidian district, Beijing, and China Agricultural University, Beijing, respectively. Both vineyards were located on sandy loam soil and received routine care suitable for commercial grape production, including pruning, fertilization, irrigation, and pest control. The 'Kyoho' and 'Seedless Wuhehong' vines were 9 and 6 years old, respectively, 


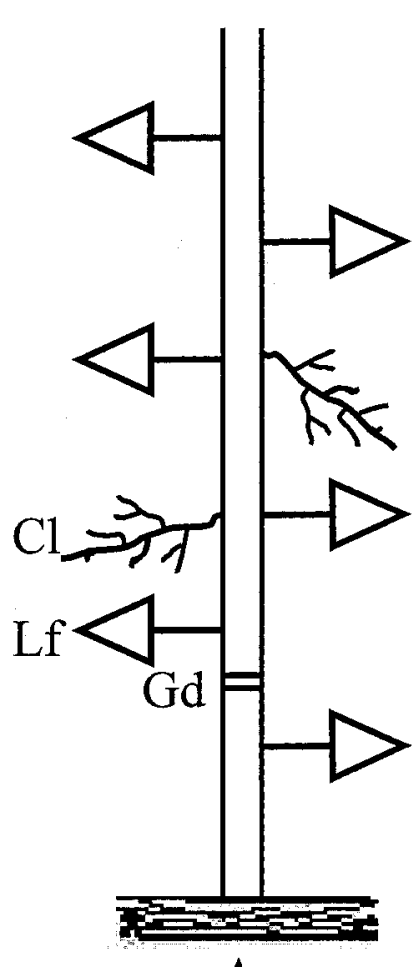

A

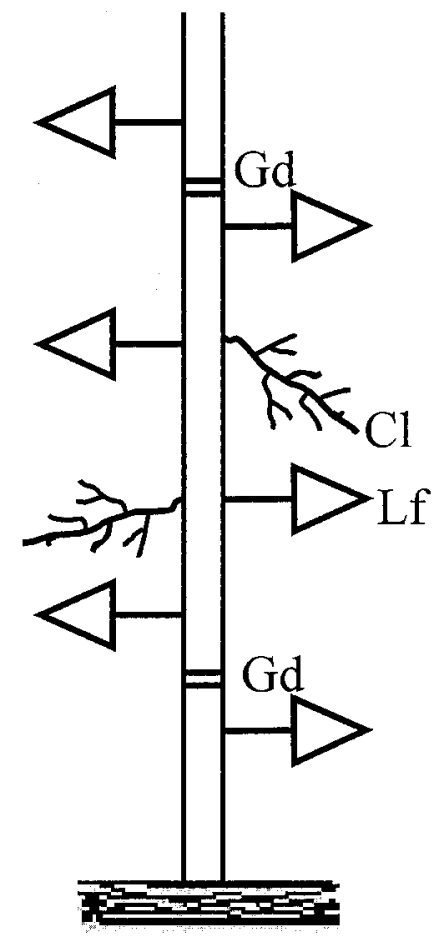

$\mathrm{B}$
Fig. 1. Shoot girdling treatment (diagram) on 'Kyoho' grapevines. (A) Single shoot girdling. (B). Double shoot girdling. $\mathrm{Lf}=$ leaf, $\mathrm{Cl}=$ grape cluster, $\mathrm{Gd}=$ girdle.

and were trained on a double vertical trellis with a fan system widely used in commercial vineyards in China. Grape clusters of the two cultivars reached full bloom $\approx 23$ May. The berries of 'Kyoho' began to soften at $47 \mathrm{~d}$ and 'Seedless Wuhehong' at 42 $\mathrm{d}$ after full bloom (DAFB).

Treatments. Two hundred thirty 'Kyoho' vines were used in this study. Two fruiting shoots were usually chosen from each vine and a total of 450 fruiting shoots having uniform orientation, vigor, and diameters of 0.95 to $1.05 \mathrm{~cm}$ at the third basal node during full bloom were selected for study. Each shoot was thinned to two basal clusters. The experiment included three blocks in a completely randomized block design with three treatments in each block. Thus, each treatment consisted of 150 fruiting shoots, with 50 shoots for each of three replications. Treatment A consisted of shoot girdling between the second and third nodes below the basal cluster 21 DAFB (Fig. 1A). The girdle removed a 4-mm strip of bark. About $80 \%$ of the girdles became well healed with a complete callus covering before 45 DAFB. Treatment B consisted of two girdlings, performed the same way and at the same time as in treatment $\mathrm{A}$, between the second and third nodes below the basal cluster and the second and third nodes above the apex cluster 21 DAFB (Fig. 1B). Girdle wounds were wrapped with clear plastic 39DAFB and became sufficiently healed in $7 \mathrm{~d}$ after wrapping. Treatment $\mathrm{C}$ was a nongirdled control.

Ninety uniform fruiting shoots with diameters of 0.7 to 0.9 $\mathrm{cm}$ were selected from 45 vines of 'Seedless Wuhehong' with two fruiting shoots on each vine. Each shoot was thinned to two clusters. Three treatments as described above were applied 21 DAFB to plots containing 30 shoots with 10 shoots for each treatment in a completely randomized block design with three blocks. All the girdles were fully healed by 45 DAFB.

SAMPLING. Sixty-seven berries from each treatment in each replication were randomly sampled between 0800 and $0900 \mathrm{HR}$ at 3- to 4-d intervals during berry development. From these ber- ries, 34 were chosen for recording the fresh weight. Seeds were removed from these berries and their fresh weight was measured. The pericarp consisting of skin and flesh (hereafter referred to as flesh) and seed samples were dried at 70 to $80^{\circ} \mathrm{C}$ until a constant weight was obtained and the percent moisture calculated. The remaining berries were used for analysis of soluble solids (SS), titratable acidity (TA), and pectic substances (PS). The berries used for plant hormone analysis were kept at $-80{ }^{\circ} \mathrm{C}$.

Thirty berries were randomly sampled from control vines of 'Seedless Wuhehong' between 0800 and $0900 \mathrm{hr}$ at 3- to 4-d intervals and their fresh weights and diameters were measured. The relationship between berry fresh weight and diameter was determined by multiple regression analysis as berry fresh weight $=0.6546 \times$ diameter $^{2.8868}(r=0.9997)$. Twelve berries from each replication ( 36 berries for each treatment) were marked after fruit set, their diameters were measured repeatedly during development at 3-d intervals using a vernier caliper. Berry fresh weight was calculated from the regression formula above and the growth kinetics of intact berries was determined. Additionally, 17 to 34 berries from 'Seedless Wuhehong' were randomly collected from each replicate plot (50 to 100 berries for each treatment) at 4- to 5-d intervals for SS, TA and PS analysis.

BioCHEMICAL ANALYSIS. Each berry sample was homogenized in a Waring commercial blender and the pulp centrifuged (1000 $\left.g_{\mathrm{n}}\right)$ to separate the supernatant, which was used for TA, SS, and PS analyses. Titratable acidity was measured by titrating 5 $\mathrm{mL}$ supernatant against $0.1 \mathrm{~N} \mathrm{NaOH}$ to an end point of $\mathrm{pH}$ 8.2. Soluble solids were determined using a hand held refractometer (WYT-76, China).

Pectin substances were extracted and quantitatively determined as described by Yang et al. (1993). Five grams of homogenate was boiled in $75 \mathrm{~mL} 95 \%$ ethanol for 30 min to remove simple sugars and phenolic compounds. The homogenate was centrifuged at $1000 g_{\mathrm{n}}$ for $15 \mathrm{~min}$ and then vacuum filtered with Whatman No.1 filter paper. The precipitate was collected and incubated in a water bath at $50{ }^{\circ} \mathrm{C}$ for $30 \mathrm{~min}$. The solution was then centrifuged and the supernatant collected for analysis of water-soluble pectin. The precipitate was suspended in $1.0 \mathrm{~N} \mathrm{H}_{2} \mathrm{SO}_{4}$ and boiled for 1 $\mathrm{h}$ to dehydrate the protopectin (water-insoluble pectin). After centrifugation, the supernatant was analyzed for galacturonic acid by colorimetric determination (Yang et al., 1993). Total pectic content was calculated as the sum of the water-soluble and water-insoluble fractions.

Analysis of Phytohormones. Methods for extraction and analysis of plant hormones were modified from Cawthon and Morris (1982). Deseeded frozen flesh and seeds of 25 and $5 \mathrm{~g}$ fresh weight, respectively, were ground to powder in liquid nitrogen, and then homogenized in 2 volumes of $100 \%$ methanol. The homogenate was mixed with 4 more volumes of cold methanol and shaken for $4 \mathrm{~h}$ at 1 to $4{ }^{\circ} \mathrm{C}$. The samples were clarified by centrifugation (15,000 $g_{\mathrm{n}}$ for $20 \mathrm{~min}, \mathrm{JA} 24$, Beckman, Beckman Instrument Inc., Palo Alto, Calif.). After centrifugation, the precipitate was incubated in $50 \mathrm{~mL}$ cold methanol overnight in a refrigerator, and then shaken for $2 \mathrm{~h}$ at 1 to $4{ }^{\circ} \mathrm{C}$ and filtered. The supernatants were pooled and condensed to $5 \mathrm{~mL}$ by means of low-pressure evaporation. Concentrations of IAA, $\mathrm{GA}_{3}$, zeatin $(\mathrm{Z})$, zeatin riboside $(\mathrm{ZR})$ and $\mathrm{ABA}$ were determined using a Waters 244 high-pressure liquid chromatograph (Waters 244) equipped with a UV detector. The hormones were separated isocratically in $25 \% \mathrm{CH}_{3} \mathrm{OH}$ and $35 \% \mathrm{CH}_{3} \mathrm{CN}$ and $40 \% \mathrm{H}_{2} \mathrm{O}(\mathrm{pH}=3)$ at a solvent flow rate of $0.7 \mathrm{~mL} \cdot \mathrm{min}^{-1}$ using a $4.6 \times 25-\mathrm{cm}$ Novapak reverse phase column with $5 \mu \mathrm{m} \mathrm{C} 18$ silica packing. Injection 
volume was $25 \mu \mathrm{L}$ and the detecting wavelength was $254 \mathrm{~nm}$. The retention time and coinjection of authentic standards were used to determine the identities of these hormones. Peak height was related linearly to concentration and used to quantify these compounds. All hormone standards were purchased from SigmaAldrich Chemical Co.

\section{Results}

Berry fresh weight growth for 'Kyoho' exhibited a double sigmoid growth pattern (Fig. 2a). The three stages of berry growth in the control were defined by the inflection points of the growth curves and the onset of veraison. Following anthesis, the berries grew rapidly for $27 \mathrm{~d}$ (Stage I). Subsequently, fresh weight increased minimally for $\approx 20 \mathrm{~d}$ (Stage II). The transition from Stage I to Stage II was characterized by an abrupt decline in average growth rates from $105 \mathrm{mg} \cdot \mathrm{d}^{-1}$ (from 24 to 27 May) to $47.6 \mathrm{mg} \cdot \mathrm{d}^{-1}$ (from 28 to $30 \mathrm{May}$ ). The rapid growth of the berry resumed at $48 \mathrm{~d}$ (veraison), and continued until fruit harvest. Veraison is characterized by the beginning of berry softening and abrupt changes in both total SS and TA. The increase in berry fresh weight during the three stages accounted for $41.4 \%, 9.6 \%$ and $49 \%$ of the final weight. The average rates of berry growth were $\approx 100,31$, and $103 \mathrm{mg} \cdot \mathrm{d}^{-1}$ for the three stages, respectively. Shoot girdling had a significant influence on the growth of the
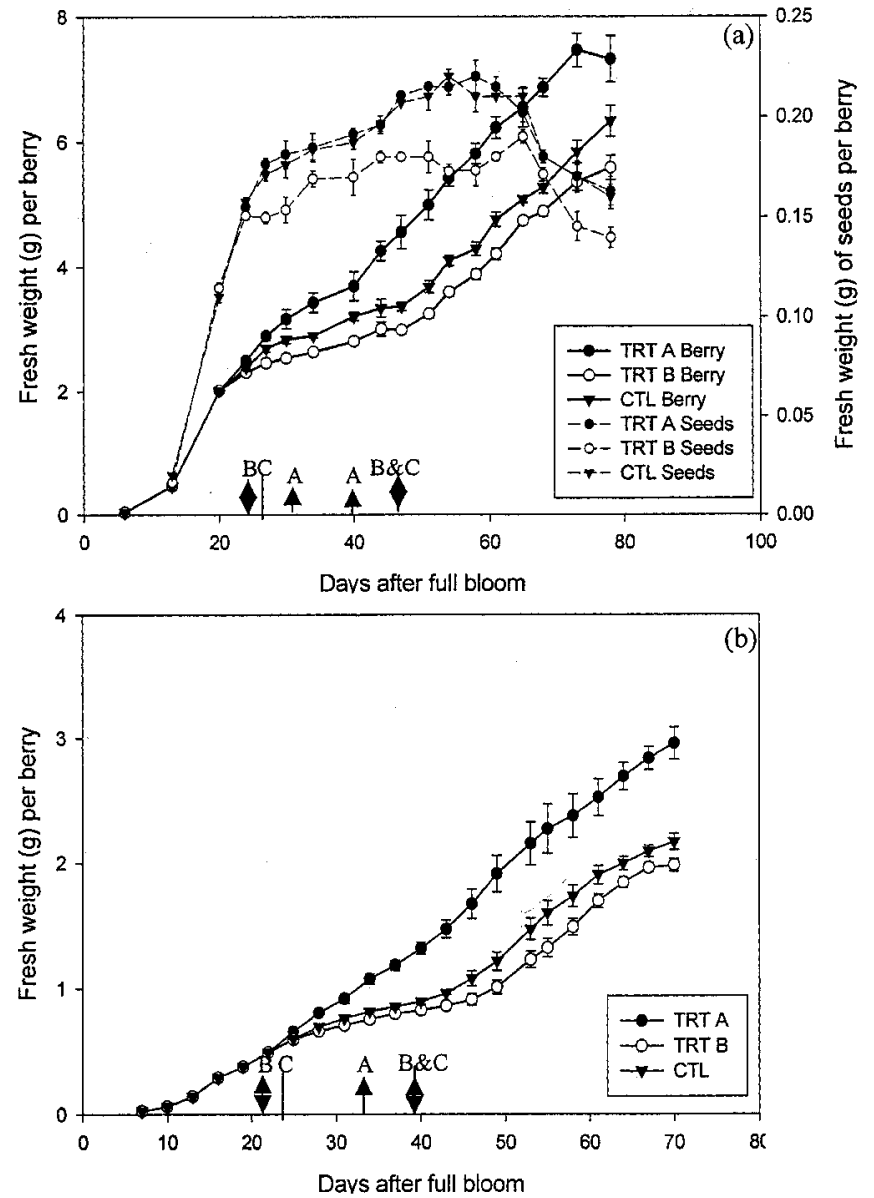

Fig. 2. Effect of shoot girdling on berry and seed fresh weight of 'Kyoho' grape (a) and on berry fresh weight of 'Seedless Wuhehong' (b). Girdling treatments A and B were applied $21 \mathrm{~d}$ after full bloom. Markers ' $\uparrow$ ', ' $\hat{\imath}$ ', '।' on the $\mathrm{x}$-axis indicate the transitions of the three stages of berry growth in treatments A, B (TRT A, and B) and C (control, CTL), respectively. Each point is a mean of three replications. Vertical bars represent \pm SE. berry. Single girdling (treatment A) extended Stage I by $3 \mathrm{~d}$ and advanced veraison (beginning of Stage III) by a week, compared with the control. Mean berry growth rates during Stage I, II and III were $106 \%, 169 \%$ and $93 \%$ those of the control. Double girdling (treatment B) advanced the beginning of Stage II by $3 \mathrm{~d}$ compared to the control. Veraison was not affected because the girdlings were healed well before Stage III. Mean growth rates during the three stages in treatment B were $96.3 \%, 95.3 \%$ and $95.2 \%$ those of the control. The double sigmoid pattern characteristic of seeded berry growth could also be clearly seen in 'Seedless Wuhehong' (Fig. 2b). Single girdling (treatment A) eliminated Stage II, resulting in a sigmoidal growth curve. Veraison was advanced by $5 \mathrm{~d}$ in this treatment compared to the control. Just as with the seeded cultivar 'Kyoho', double girdling (treatment B) advanced Stage II by $3 \mathrm{~d}$, but did not change the time of the veraison compared to the control.

Seed fresh weight exhibited sigmoidal growth pattern in 'Kyoho' (Fig. 2a) and was characterized by rapid weight increase during Stage I, reaching 93.2\% of the final weight and showing a plateau from Stage II on. Although neither girdling treatments A nor B changed the sigmoidal pattern of seed growth, the decreased seed size in treatment B was evident during the growing season.

Berry dry weight also exhibited a double sigmoidal curve in 'Kyoho' (Fig. 3a). The mean growth rates during the three
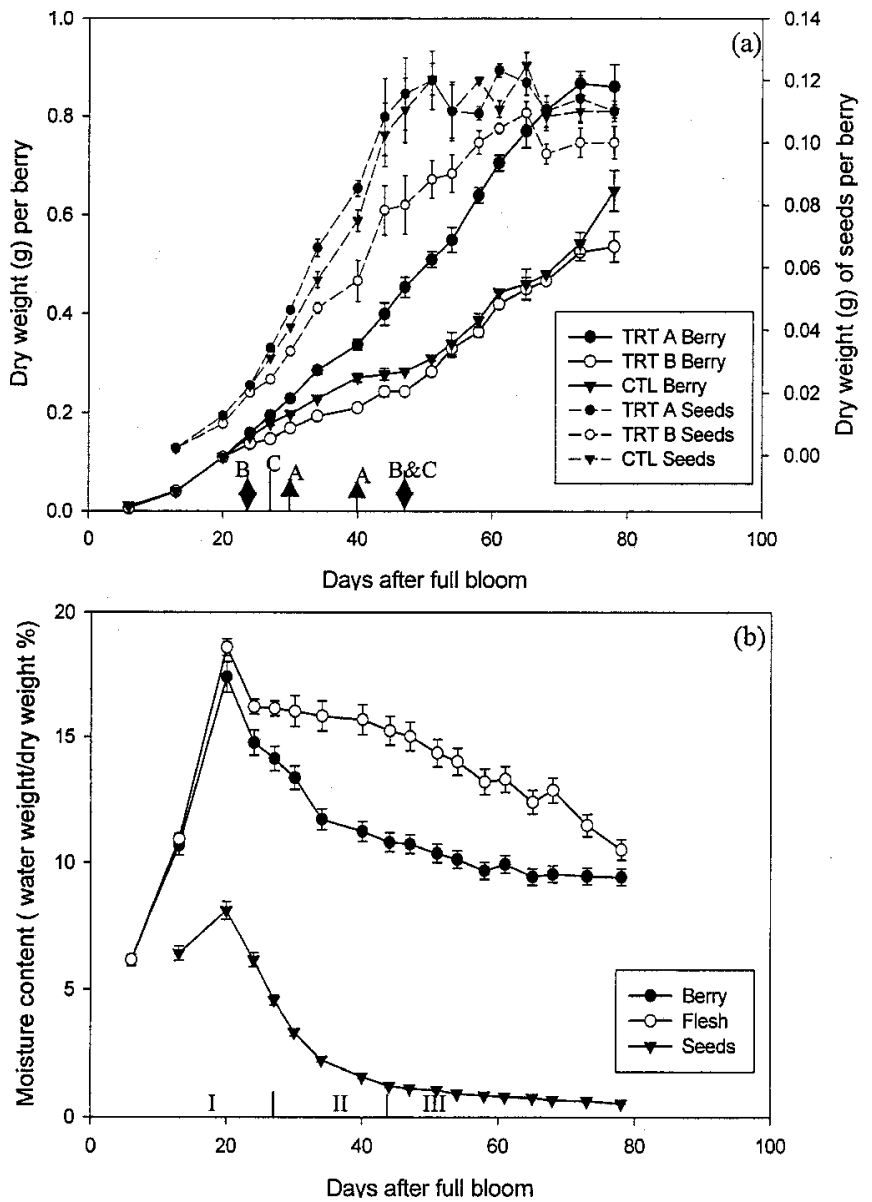

Fig. 3. Effect of shoot girdling on grape berry and seed dry weight (a) and changes in percent moisture of berry, seed, and flesh (b) of 'Kyoho' grape. Girdling treatments A and B were applied $21 \mathrm{~d}$ after full bloom. Markers ' $\uparrow$ ', ' $\uparrow$ ', 'l' on the $\mathrm{x}$-axis indicate the transition of the three stages of berry growth in treatments A, B (TRT A, and B) and C (control, CTL), respectively. Each point is a mean of three, 34-berry replications. Vertical bars represent \pm SE. 
stages of berry growth were $6.6,5.2$ and $11.8 \mathrm{mg} \cdot \mathrm{d}^{-1}$. Girdling significantly influenced the double sigmoidal pattern of berry dry weight accumulation. The growth curve of berry dry weight in treatment A followed a sigmoidal pattern without Stage II being observed. If calculated according to three growth stages of berry fresh weight change, the corresponding mean growth rates for dry weight were $7.6,11.0$ and $13.7 \mathrm{mg} \cdot \mathrm{d}^{-1}$. However, dry weight in treatment B averaged $86.6 \%, 88.4 \%$, and $80.3 \%$ as much as the control during the three stages.

The pattern of seed dry weight accumulation was sigmoidal (Fig. 3a). The most rapid rates of increase in dry weight occurred during Stages I and II, contributing 32.3\% and 58.5\%, respectively, to the maximum size at maturity. Seed dry weight reached its maximum at veraison. The dry weight of the seeds, although not significantly affected by treatment $\mathrm{A}$, declined in treatment B.

The patterns of water accumulation on a whole berry or flesh only basis were both sigmoidal, with the three stages corresponding to those of berry fresh weight (data not shown). The moisture content in seeds was highest (140 mg/berry) at the end of Stage $\mathrm{I}$, and then rapidly declined to a steady level of $60 \mathrm{mg} /$ berry at the end of Stage II. The percent moisture of the berry on a dry weight basis increased rapidly, reaching a maximum 20 DAFB (Fig. 3b). Following 20 DAFB, the moisture percentages de-
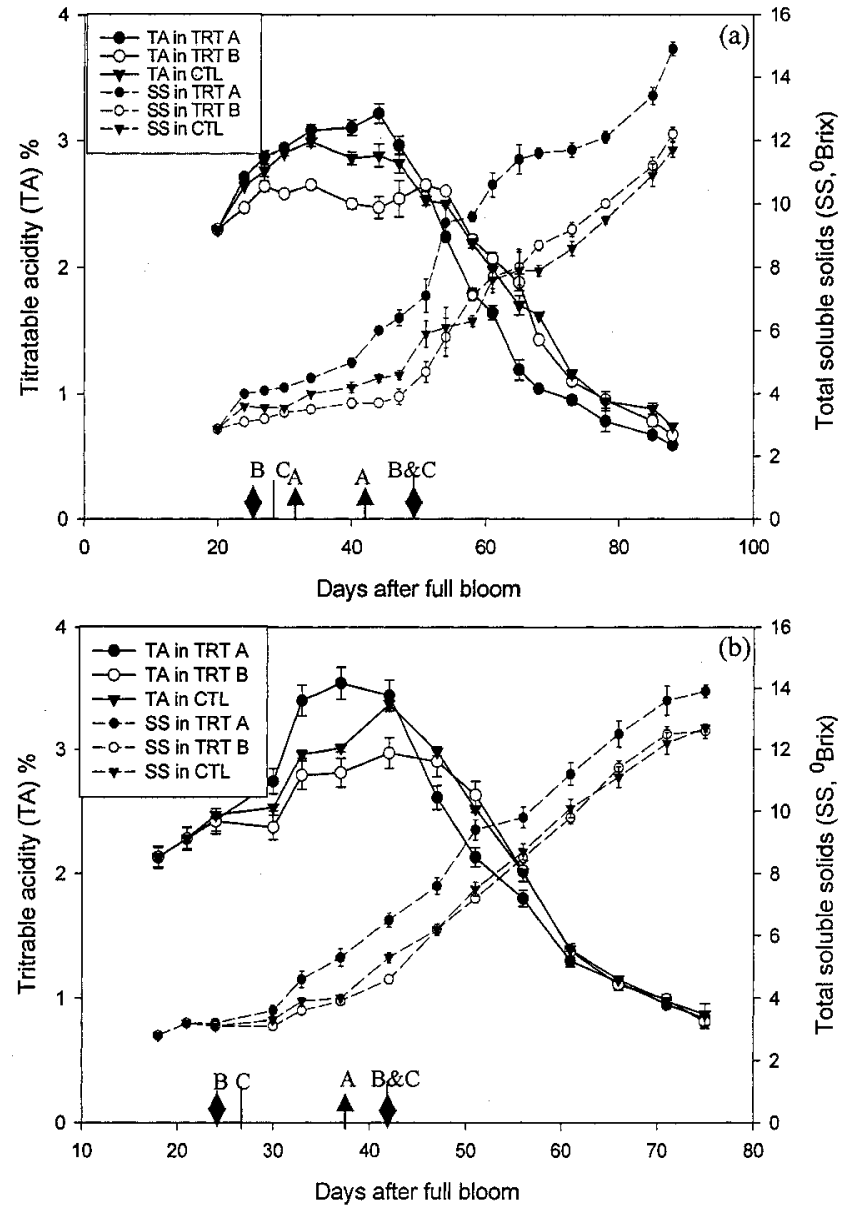

Fig. 4. Changes in soluble solids (SS) and titratable acidity (TA) in 'Kyoho'(a) and 'Seedless Wuhehong'(b) grapes in response to shoot girdling. Girdling treatments A and B were applied $21 \mathrm{~d}$ after full bloom. Markers ' $\uparrow$ ', ' $\uparrow$ ', 'l' on the $\mathrm{x}$-axis indicate the transition of the three stages of berry growth in treatments A, B (TRT A, and B) and C (control, CTL), respectively. Each point is a mean of three replications. Vertical bars represent \pm SE. creased rapidly until the middle of Stage II, and then gradually decreased until maturity.

Berry SS averaged $3^{\circ}$ Brix 20 DAFB and increased very slowly until the end of Stage II (Fig. 4a). Thereafter, soluble solids increased rapidly until 90 DAFB. The TA of the berries increased gradually until the beginning of Stage II, reaching its peak of $\approx 3 \%$ at $33 \mathrm{DAFB}$, and declining rapidly thereafter, to $0.6 \%$ at harvest. Girdling had a significant influence on the percentages of both SS and TA. The increase in SS and the decrease in TA at veraison occurred $7 \mathrm{~d}$ earlier in treatment $\mathrm{A}$ than in the control. Additionally, during Stage II of the berry growth, the accumulation of both SS and TA increased in treatment A. Treatment B did not affect the time of veraison and inhibited the accumulation of both SS and TA during the preveraison period. The patterns of SS and TA concentrations during the growth and development of 'Seedless Wuhehong' berries and the effects on them by shoot girdling (Fig. 4b), were similar to those in 'Kyoho'.

The concentration of both water-insoluble and water-soluble pectins in berries of 'Kyoho' remained relatively steady until $7 \mathrm{~d}$ after veraison (Fig. 5a). Thereafter, rapid and significant changes were observed. The concentration of water-insoluble pectin decreased from 1.9 to $0.58 \mathrm{mg} \cdot \mathrm{g}^{-1} \mathrm{FW}$ at harvest, while the concentration of water-soluble pectin increased from 0.20 to 0.49 $\mathrm{mg} \cdot \mathrm{g}^{-1} \mathrm{FW}$. In response to treatment A, there were rapid changes
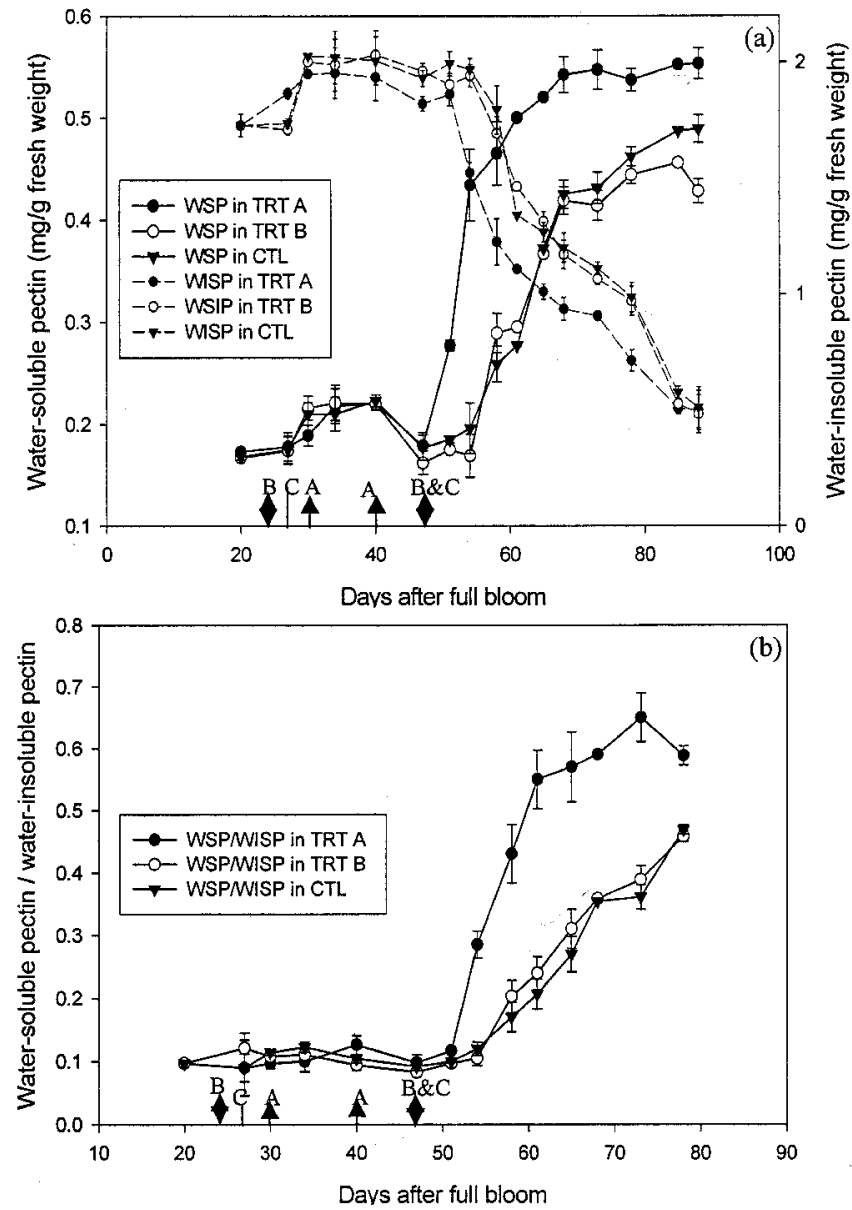

Fig. 5. Changes in the concentrations of water-soluble pectin (WSP) and waterinsoluble pectin (WISP) (a) and in the ratio of WSP to WISP (b) during the development of 'Kyoho' grape. Girdling treatments A and B were applied 21 $\mathrm{d}$ after full bloom. Markers ' $\uparrow$ ', ' $\hat{\imath}$ ', 'l' on the $\mathrm{x}$-axis indicate the transition of the three stages of berry growth in treatments A, B (TRT A, and B) and C (control, CTL), respectively. Each point is a mean of three replications. Vertical 
in both water-insoluble and water-soluble pectin 51 DAFB, which did not correspond to the period of rapid berry growth immediately after veraison (40 DAFB). In response to treatment B, the significant changes in pectin concentration were observed a week after veraison, similar to that of the control. The changes in pectic substances were also discernible from the dynamics of the ratio of water-soluble pectin/water-insoluble pectin (Fig. 5b). The ratio was $\approx 10 \%$ and constant throughout Stages I and II berry development, increasing to $58.8 \%, 45.8 \%$, and $46.9 \% 78$ DAFB in treatment A, B and the control, respectively. Changes in pectic substances in 'Seedless Wuhehong' (Fig. 6a), unlike those in 'Kyoho', corresponded to the period of rapid changes in SS and TA and to fruit growth and softening immediately after veraison. The rates of changes in pectic substances were less than those of SS, TA and berry growth since $5 \mathrm{~d}$ postveraison. From then on, rapid changes in the ratio of water-soluble/water-insoluble pectin were observed, which corresponded to the time when $50 \%$ of berries in treatment A (42 DAFB), and 30\% to $40 \%$ of berries in treatment $\mathrm{B}$ and control (47 DAFB) began softening (Fig. 6b).

The highest concentration of IAA was observed in flesh during the early portion of Stage I (Fig. 7a). IAA concentration decreased rapidly from the later part of Stage I throughout Stage II, with a relatively low concentration observed at veraison. IAA was no longer detectable two weeks after veraison. Girdling had little
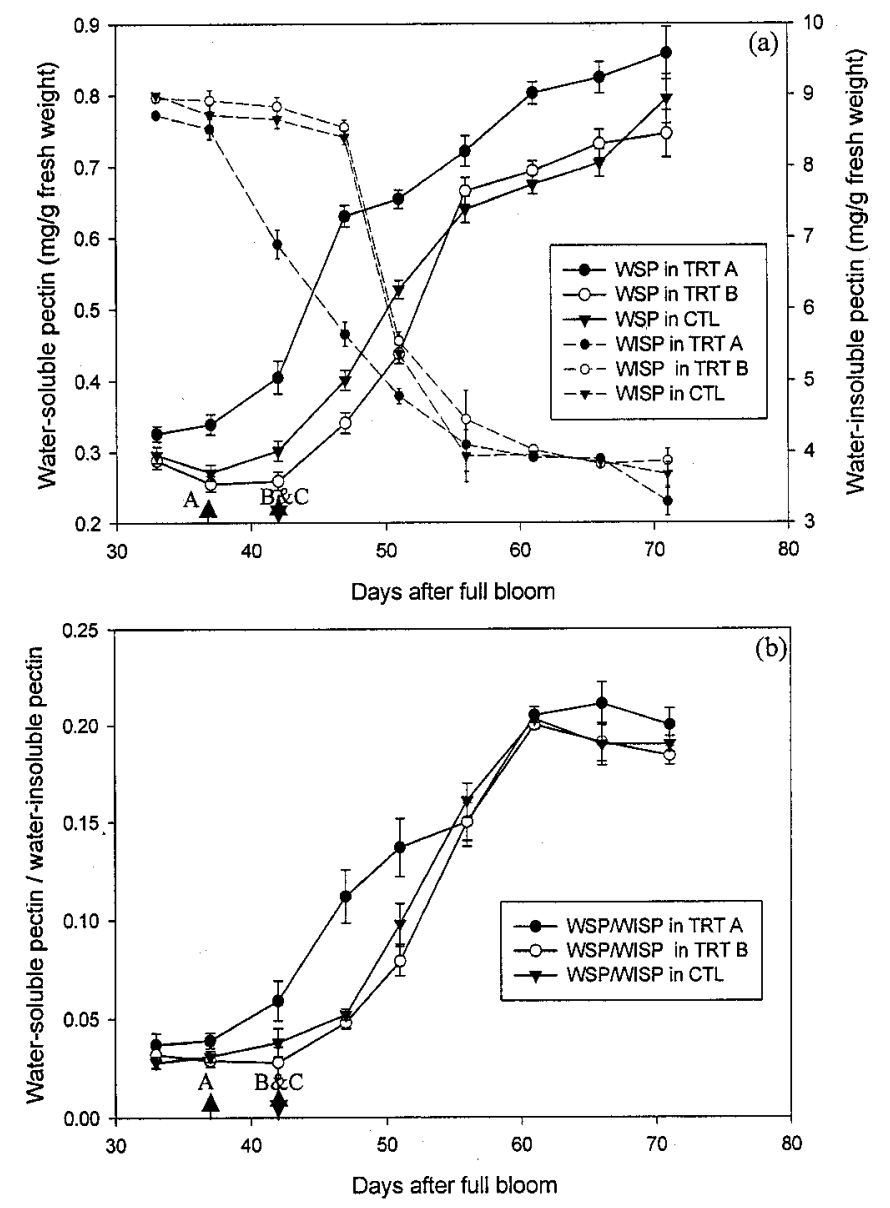

Fig. 6. Changes in the concentrations of water-soluble pectin (WSP) and waterinsoluble pectin (WISP) (a) and in the ratio of WSP to WISP (b) during the development of 'Seedless Wuhehong'. Girdling treatments Aand B were applied $21 \mathrm{~d}$ after full bloom. Markers ' $\uparrow$ ', ' $\uparrow$ ', and 'I' on the $\mathrm{x}$-axis indicate the veraison in treatments A, B (TRT A, B) and C (control, CTL), respectively. Each point is a mean of three replications. Vertical bars represent \pm SE. influence on the general pattern of changes in endogenous IAA, with the exception of a transient decrease $4 \mathrm{~d}$ after girdling in treatment $\mathrm{A}$ and a transient increase $4 \mathrm{~d}$ after girdling in treatment $B$. The concentration of IAA in seeds was higher than that in the flesh and rose rapidly throughout Stage I, peaking 30 DAFB, when the rapid growth and development of seeds occurred (Fig. 2a). This was followed by a rapid decline in IAA in the middle of Stage II, and by two weeks after veraison, IAA was nondetectable. The IAA concentrations were similar among girdling treatments and the control.

The level of $\mathrm{GA}_{3}$ was high in the flesh at Stage I and gradually declined throughout Stage II and Stage III (Fig. 7b). The decrease in $\mathrm{GA}_{3}$ concentration was faster in treatment $\mathrm{A}$ than in the control, however, the $\mathrm{GA}_{3}$ concentration steadily increased in treatment $\mathrm{B}$ before a rapid decline during Stage II. $\mathrm{GA}_{3}$ concentrations were similar among treatments from 30 DAFB throughout ripening. Just as with IAA, $\mathrm{GA}_{3}$ concentration was much higher in the seeds than in the flesh (Fig. 7b). Seed $\mathrm{GA}_{3}$ concentration was constant early in the season, and began to increase rapidly at early Stage II. The concentration peaked at $40 \mathrm{DAFB}$, the period when rapid development and differentiation of the embryo and endosperm occur. The $\mathrm{GA}_{3}$ concentration decreased rapidly before veraison, and remained low after veraison. Girdling had no influence on the concentration of $\mathrm{GA}_{3}$ in seeds.
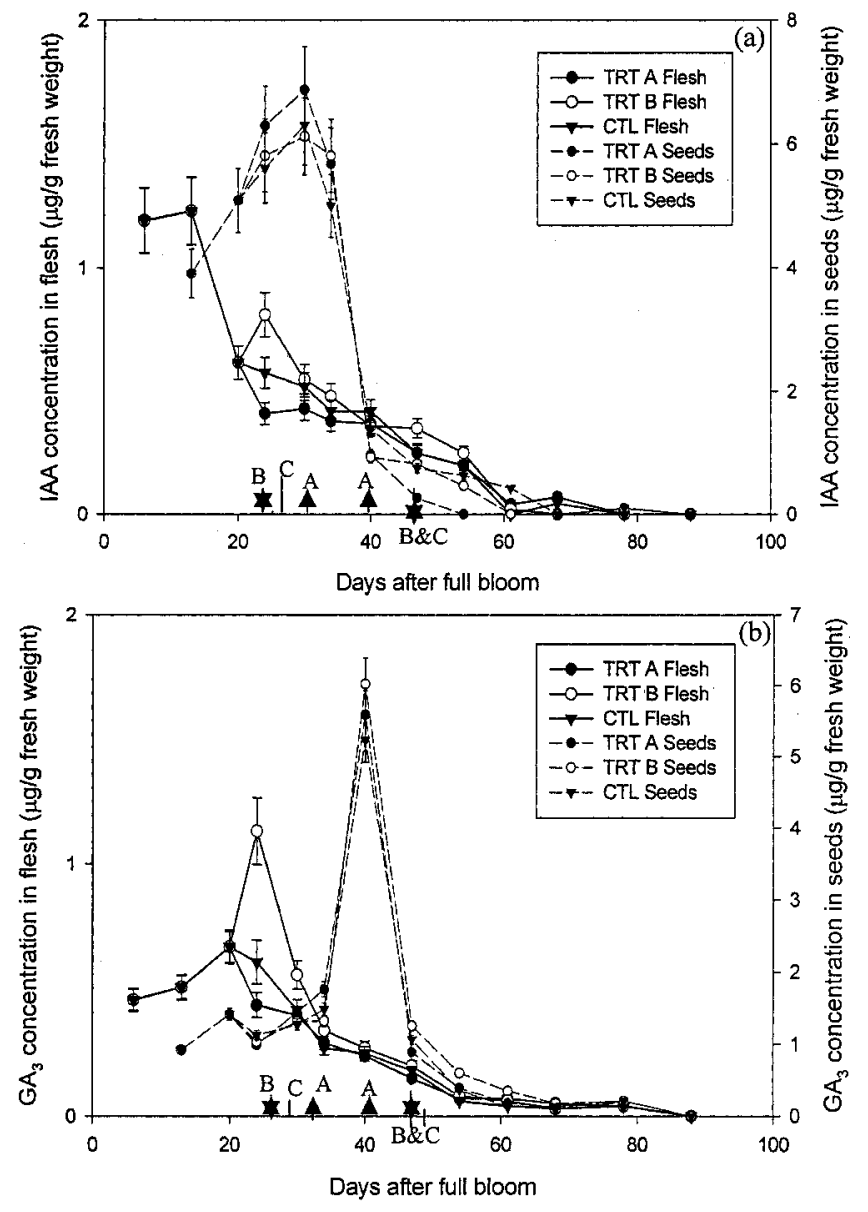

Fig. 7. Changes in the concentration of indoleacetic acid (IAA) (a) and gibberellic acid $\left(\mathrm{GA}_{3}\right)(\mathbf{b})$ in the flesh and seeds of 'Kyoho' grape in response to shoot girdling. Girdling treatments A and B were applied $21 \mathrm{~d}$ after full bloom. Markers ' $\uparrow$ ', ' $\uparrow$ ', ' $I$ ' on the $\mathrm{X}$-axis indicate the transition of the three stages of berry growth in treatments A, B (TRT A, and B) and C (control, CTL), respectively. Each point is a mean of three replications. Vertical bars represent \pm SE. 
Zeatin concentrations were high in the flesh one week after full bloom, but rapidly declined to undetectable levels after veraison (Fig. 8a). Girdling treatments did not alter zeatin concentration. Zeatin concentration in the seeds increased rapidly beginning 20 DAFB, reaching its peak 34 DAFB, in the middle of Stage II of berry development. Zeatin was significantly higher in treatment $\mathrm{B}$ than in treatment $\mathrm{A}$ and the control at 34 DAFB. The concentration of zeatin riboside in the flesh was high during the early season, showing a peak 24 DAFB, but declined rapidly by the beginning of Stage II (Fig. 8b). Girdling had no influence on the concentration of zeatin riboside in the flesh. The concentration of zeatin riboside in seeds followed a bell-shaped curve with a peak at 40 DAFB (Fig. 8b). Girdling increased (Treatment B) or decreased the (Treatment A) zeatin riboside concentration at 40 DAFB.

The highest concentration of ABA was observed in the flesh in the early season, followed by a continuous and rapid decline (Fig. 9). ABA was nondetectable 40 DAFB, but increased two weeks after veraison. ABA was detected two and seven weeks after veraison in treatments Aand B, respectively. ABAconcentration in the seeds was relatively high in Stage I, but decreased gradually to undetectable levels in the middle of Stage II (Fig. 9). ABA concentration rose again two weeks after veraison. ABA concentrations also increased 2 weeks after veraison in both treatments A and B.
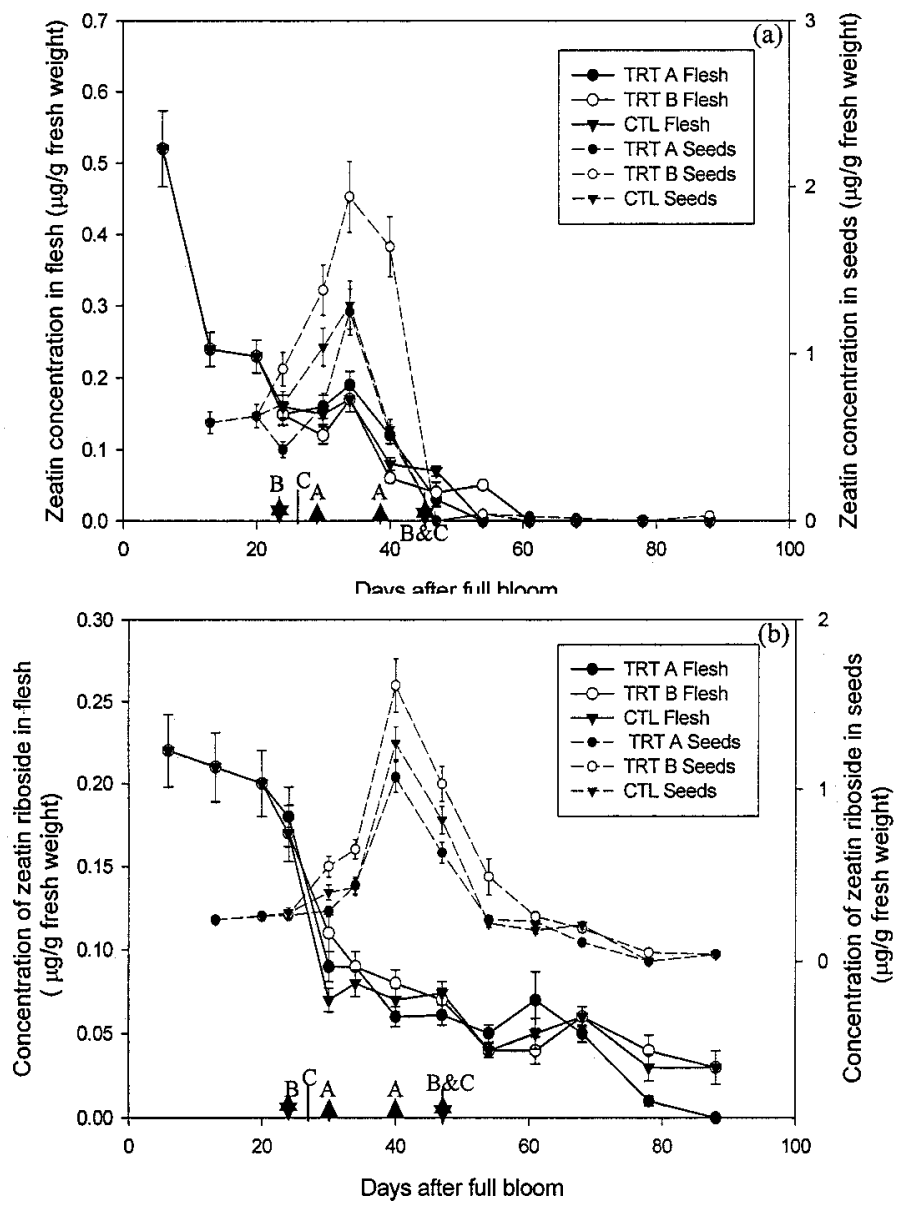

Fig. 8. Changes in the concentration of zeatin (Z) (a) and zeatin riboside (ZR) (b) in the flesh and seeds of 'Kyoho' grape in response to shoot girdling. Girdling treatments A and B were applied $21 \mathrm{~d}$ after full bloom. Markers ' $\uparrow$ ', ' $\imath$ ', 'I' on the $\mathrm{x}$-axis indicate the transition of the three stages of berry growth in treatments A, B (TRT A, and B) and C (control, CTL), respectively. Each point is a mean of three replications. Vertical bars represent \pm SE.

\section{Discussion}

Our results showed a marked decline in growth rate (both fresh and dry weight) during Stage II of grape berry growth. This suggests that the berry was a relatively weaker sink during this period compared with Stages I and III. Matsui et al. (1985) observed that a significantly larger amount of ${ }^{14} \mathrm{C}$ was translocated into berries at Stages I and III compared with Stage II. Girdling in grapevines has been reported to increase berry weight and advance fruit maturity by altering assimilate translocation and distribution (Harrel and Williams, 1987; Zabadal, 1992). In our experiment, a single girdling in the later portion of Stage I presumably interrupted the assimilate translocation out of the girdled shoot, resulting in enhanced assimilate supply to the berry, although this was not actually measured. Concomitant with this alteration in assimilate distribution was a decrease in the length of Stage II. Double girdlings as in treatment B presumably prevented assimilate translocation into grape clusters between girdles, resulting in decreased assimilate supply and this was accompanied by an advance in the onset of Stage II and a decrease in berry growth rate during this period. These observations suggest that the assimilate supply plays a critical role in regulating the growth stages of grape berry.

Assimilate competition also existed within the berry. While the rate of dry weight accumulation of berry flesh gradually declined during Stage II, the dry weight of the seeds rapidly increased, suggesting the seeds were the stronger sink during this period. Many reports (Harrel and Williams, 1987; Wang and Luo, 1996; $\mathrm{Xu}$ et al., 1995) have shown that Stage II is marked by rapid respiration, growth and differentiation of the embryo and active nutrient storage and lignification in the seed, all of which require carbohydrates, lipids and proteins. In our experiment, increasing assimilate supply by a single girdling appeared to satisfy the assimilate requirements for growth and development of seed, and also to stimulate the accumulation of SS and TA in the flesh. This could reduce the osmotic and water potentials and, therefore, enhance water uptake into vacuole. Together, this would account for the increased fresh and dry weight accumulation during Stage II observed in response to treatment A compared with the control in 'Kyoho'. The assimilate competition within the berries

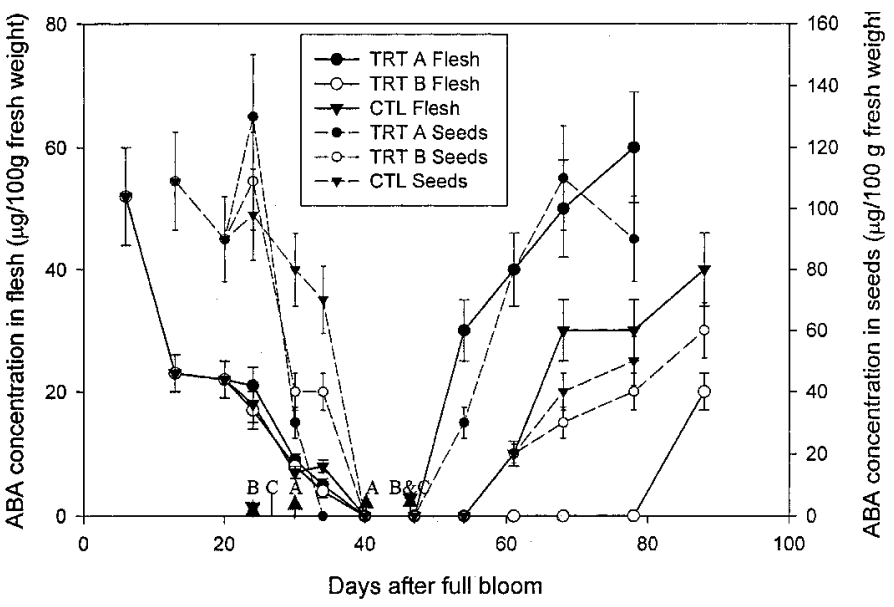

Fig. 9. Changes in the concentration of abscisic acid (ABA) in the flesh and seeds of 'Kyoho' grape in response to shoot girdling. Girdling treatments A and B were applied $21 \mathrm{~d}$ after full bloom. Markers ' $\uparrow$ ', ' $\uparrow$ ', 'I' on the $\mathrm{x}$-axis indicate the transition of the three stages of berry growth in treatments A, B (TRT A, and B) and C (control, CTL), respectively. Each point is a mean of three, 33-berry replications. Vertical bars represent \pm SE. 
was also observed in 'Seedless Wuhehong', in which Stage II disappeared after increased assimilate supply was provided by a single girdling. On the other hand, decreasing assimilate supply by double girdlings increased the assimilate competition between flesh and seed, advanced the onset of the Stage II, and reduced the growth rate of both flesh and seed.

An additional possible explanation for the decreased growth in Stage II may involve changes in chemical constituents in the berry. In Stage I, a large quantity of water-soluble substances accumulated within the berry, increasing water uptake and in turn causing a rapid increase in the fresh weight of the berry (both flesh and seed). Beginning at the end of Stage I until veraison, not only did the accumulation rate of berry dry matter gradually decline, but also the percent moisture rapidly decreased. These were correlated with a decrease in berry growth rate. According to Xu et al. (1995), and Wang and Luo (1996), a large amount of highly hydrophobic lipids and some proteins are stored within seeds, and structural polysaccharides such as cellulose and lignin are condensed into spermodermis (seed coat). The moisture percentage may have decreased rapidly when soluble solids and metabolites were converted into these hydrophobic substances. Slow berry growth during Stage II may be the result of a decrease in the rate of water accumulation on a berry basis, and a decrease in accumulation of dry matter in the berry due to assimilate competition between the berry and the grapevine (Novello et al., 1999), and within the berry itself (i.e., flesh and seed).

Changes in endogenous plant hormones have been demonstrated in whole grape berries during development (Cawthon and Morris, 1982; Inaba et al., 1976; Niimi et al., 1977; Tromp, 1989; Xu et al., 1995). In our study, endogenous growth substances were investigated separately in the flesh and seeds. Levels of growthstimulating hormones such as IAA, $\mathrm{GA}_{3}$ and $\mathrm{ZR}$ were quite high in the flesh during the early stage of berry development, while ABA concentrations rapidly decreased during the same period. The results concur well with previous observations (Cawthon and Morris, 1982; Inaba et al., 1976; Niimi et al., 1977; Pandey and Singh, 1989), and suggest that the changes may be related to the first rapid growth phase of the berry for enhancing cell division or enlargement, possibly due to the regulation of assimilate partitioning within the grapevine (Brenner et al., 1989). The concentrations of IAA, $\mathrm{GA}_{3}, \mathrm{Z}$ and $\mathrm{ZR}$ in flesh gradually declined to very low levels during the middle of Stage II. On the other hand, IAA concentrations in seeds maintained high levels, while $\mathrm{GA}_{3}, \mathrm{Z}$ and ZR also increased rapidly and reached their peaks in the seeds during Stage II of berry growth. These observations matched with previous reports (Cawthon and Morris, 1982; Inaba et al., 1976). The peak shifts of growth-promoting hormones like IAA, $\mathrm{GA}_{3}$ and cytokinin between flesh and seeds suggested that the active center of the sink within berries shifted from the flesh to the seeds during Stage II, when the rapid growth and development of seeds occurred. High concentrations of IAA, GA 3 , Z, and ZR in seeds may have promoted the translocation of assimilates to the seeds in order to maintain development of the embryo and storage of nutrients in the endosperm (Brenner et al., 1989). The changes in $\mathrm{Z}$ and ZR peak levels in seeds during Stage II in response to shoot girdlings suggest the existence of feedback responses to accumulation of these hormones in seeds by varying levels of assimilate supply during Stage II of berry growth. Niimi et al. (1977) and Xu et al. (1995) hypothesized that a high concentration of ABA observed during Stage II was responsible for the slow berry growth. In our study, however, no peak of ABA was found at that time. The decline in ABA concentration found in both the flesh and the seed was similar to observations by Ohkawa (1985) and Panday and Singh (1989). These results suggest that ABA may not be responsible for the slow berry growth during Stage II. On the contrary, low levels of ABA might be closely related to the rapid growth and development of the seeds. Low concentrations of ABA in the flesh suggest that there might be other growth inhibitors such as phenolic compounds, which decrease the growth of the flesh (Ohkawa, 1985).

The initiation of veraison completes Stage II. In our study, low concentrations or the disappearance of $\mathrm{GA}_{3}, \mathrm{Z}$, and $\mathrm{ZR}$ observed in both flesh and seeds suggest that they are not closely related to the second active growth phase (Stage III) of the berry. This concept is supported by a previous report that exogenous $\mathrm{GA}_{3}$ and cytokinin had almost no effect on the ripening of 'Delaware' grapes (Inaba et al., 1976). Several studies have reported that fruit ripening does not commence until ABA increases in the berry tissue (Cawthon and Morris, 1982; Inaba et al., 1976; Niimi et al., 1977), and exogenous ABA hastens ripening (Inaba et al., 1976), suggesting that ABA is the direct trigger of veraison in the grape berry. In our experiment, however, increases in ABA concentration, either in flesh or in seeds, occurred one to four weeks after veraison in all treatments. Our results showed that there was not a strong correlation between veraison and ABA changes, suggesting that ABA may not be the only trigger for veraison in the grape berry and may play a role in stimulating nutrient accumulation (Ndung'u et al., 1996) and enhancing the berry ripening during late Stage III (Shiozaki et al., 1999). Our findings emphasize the necessity of further research in this area. Much attention has also been paid to the relationship of initiation of fruit ripening and IAA, which was high during the early stages of berry development, but declined prior to the initiation of ripening (Cawthon and Morris, 1982; Niimi et al., 1977). Exogenous auxin was reported to delay the berry ripening process (Inaba et al., 1976). In our experiment, the low levels of IAA in both seeds and flesh at veraison coincide with the cessation of seed development and the decrease in sink activity of the seeds.

The increase in the proportion of water-soluble pectin to total pectin (or to water-insoluble pectin), resulting from degrading of pectic substances, occurred simultaneously with berry softening and rapid changes in SS and TA in 'Seedless Wuhehong'. Silacci and Morrison (1990) also reported a substantial increase in soluble pectin in 'Cabernet Sauvignon'. These results suggested that changes from veraison onward in the pectic content of grapes play an important role in fruit softening. However, in our experiment with 'Kyoho', we did not find the changes in watersoluble and water-insoluble pectin to be associated with other physical and chemical changes at veraison. Changes in pectic substances in the berry of 'Kyoho' lagged behind those of SS and TA, and behind the onset of berry softening at veraison. Similar results were also observed by the authors in 'Muscat Hamburg', in which the content of pectin and ratio of two proportions in the berry did not change until $10 \mathrm{~d}$ post-veraison, when $70 \%$ of berries were undergoing softening (Zhang, unpublished data). Our results suggest that changes in pectic substances stimulated the softening process in late Stage III, but were not closely related to the onset of softening at veraison in 'Kyoho'. Changes in other cell wall constituents, such as cellulose, might be responsible for the softening process in early Stage III. Our results were also supported by Robertson et al. (1980) who found no increase in water-soluble pectin during ripening. Therefore, different softening mechanisms likely exist in Stage III of the development of berries of different grape cultivars. 


\section{Literature Cited}

Brenner, M.L., B.M.N. Schreiber, and R.J. Jones. 1989. Hormonal control of assimilate partitioning: Regulation in the sink. ISHS Acta Hort. 239:141-148.

Cawthon, D.L. and J.R. Morris. 1982. Relationship of seed number and maturity to berry development, fruit maturation, hormonal changes and uneven ripening of Concord grapes. J. Amer. Soc. Hort. Sci. 107: 1097-1104.

Coombe, B.C. 1976. Development of the fleshy berry. Annu. Rev. Plant Physiol. 27:507-528.

Coombe, B.C. 1989. The grape berry as a sink. ISHS Acta Hort.. 239: 149-158.

Coombe, B.C. 1992. Research on development and ripening of the grape berry. Amer. J. Enol. Viticult. 43:101-110.

Coombe, B.C., M. Bovio, and A. Schneider. 1987. Solute accumulation by grape pericarp cells. J. Expt. Bot. 38:1789-1798.

Geza, H., G.F. Parsons, and L.R. Mattick. 1984. Physiological and biochemical events during development and maturation of grape berries. Amer. J. Enol. Viticult. 35:220-227.

Harrel, C.D. and L.E. Williams. 1987. Net $\mathrm{CO}_{2}$ assimilation rate of grapevine leaves in response to trunk girding and gibberellic acid application. Plant Physiol. 83:457-459.

Inaba, A., M. Ishidia, and Y. Sobajima. 1976. Changes in endogenous hormone concentrations during berry development in relation to the ripening of Delaware grapes. J. Jpn. Soc. Hort. Sci. 45(3):245-252.

Matsui, H., E. Yuda, S. Nakagawa, and K. Imai. 1985. The translocation and distribution of photosynthates in Delaware grapes. J. Jpn. Soc. Hort. Sci. 54:184-191.

Niimi, R., M. Ohkawa, and H. Torikata. 1977. Changes in auxin and abscisic-like activities in grape berries. J. Jpn. Soc. Hort. Sci. 46: 139-144.

Niimi, Y. and H. Torikata. 1979. Changes in photosynthesis and respiration during berry development in relation to the ripening of Delaware grapes. J. Japan. Soc. Hort. Sci. 47:448-453.

Ndung'u,C.K., M. Shimizu, G. Okamoto, and K. Hirano. 1996. Changes in abscisic acid, carbohydrates and nitrogenous compound of "Riesling" grapevines during induction of second shoots by water-deficit stress. Environ. Control Biol. 34:115-122.

Novello,V., L.D.E. Palma, and L. Tarricone. 1999. Influence of cane girdling and plastic covering on leaf gas exchange, water potential and viticultural performance of table grape cv. Matilde. Vitis 38:51-54.

Ohkawa, M. 1985. The relationship between growth inhibitor and phenols in developing berry of Muscat of Alexandria grape. J. Jpn. Soc. Hort. Sci. 53:383-389.

Pandey, S.N. and R. Singh. 1989. Endogenous level of hormones in developing grape seed (Vitis vinifera L.). India J. Plant Physiol. 32: 299-305.

Peruzzo, E.L. 1994. Ringing of canes in table grape cultivars. Agropecuaria Catarinense. 7(4):21-23.

Robertson, G.L., R. Eschenbruch, and K.J. Creswell. 1980. Seasonal change in the pectin substances of grapes and their implication in juice extraction. Amer. J. Enol. Viticult. 31:162-164.

Silacci, M.W. and J.C. Morrison. 1990. Changes in pectin content of Cabernet Sauvignon grape berries during maturation. Amer. J. Enol. Viticult. 42:111-115.

Shiozaki, S., Y. Kamata, T. Ogata, S. Horiuchi, and K. Kawase. 1999. Localization of abscisic acid in grape berry by immunohistochemical techniques. J. Jpn. Soc. Hort. Sci. 68:1-9.

Staudt, G., W. Schneider, and J. Leidel. 1986. Phases of berry growth in Vitis vinifera. Ann. Bot. 58:789-800.

Tromp, J. 1989. Interrelationships between plant growth regulators and fruit tree mineral nutrition. ISHS Acta Hort. 239:399-408.

Wang, J. and G. Luo. 1996. The Embryo and endersperm development in 'Kyoho' grapevines. Acta Hort. Sinica 23(2):191-193.

Xu, X., G. Luo, and Y. Peng. 1995. Dynamics and characterics of growth and development of grape berry in 'Muscat Hamburg'. Acta Hort. Sinica 22:318-322.

Yang, D., J. Dai, X. Pang, H. Xing, and L. Wang. 1993. Changes of polygalacturonase, pectin, and ultrastructure of cell wall in kiwifruit during senescence. Acta Hort. Sinica 20:341-345.

Zabadal, T.J. 1992. Response of 'Himrod' grapevines to cane girdling. HortScience 27(9):975-976. 